\title{
Adipose-Derived Stem Cells Promote Proliferation and Invasion in Cervical Cancer by Targeting the HGF/c-MET Pathway
}

This article was published in the following Dove Press journal: Cancer Management and Research

\author{
Yongning Zhai ${ }^{1,2, *}$ \\ Wangfei $\mathrm{Wu}^{3, *}$ \\ Xiaowei $\mathrm{Xi}^{4}$ \\ Rongbin $\mathrm{Yu}^{\prime}$
}

'Department of Epidemiology, School of Public Health, Nanjing Medical University, Nanjing 21 II66, People's Republic of China; ${ }^{2}$ Department of Gynecology, Women's Hospital of Nanjing Medical University, Nanjing Maternity and Child Health Care Hospital, Nanjing 210004, People's Republic of China; ${ }^{3}$ Department of Pathology, Women's Hospital of Nanjing Medical University, Nanjing Maternity and Child Health Care Hospital, Nanjing 210004, People's Republic of China; ${ }^{4}$ Department of Obstetrics and Gynecology, Shanghai General Hospital of Nanjing Medical University, Shanghai 200080, People's Republic of China

*These authors contributed equally to this work
Background: Cervical cancer is a serious female malignancy affecting women's health worldwide. The HGF/c-MET signaling pathway is activated in cervical cancer. Adiposederived stem cells (ADSCs) with multipotential differentiation can carry out paracrine secretion of hepatocyte growth factor (HGF). Here, we investigated the effect and underlying mechanism of ADSCs on the promotion and invasion of cervical cancer in vitro and in vivo.

Materials and Methods: ADSCs were isolated, identified, and co-cultured with cervical cancer cells. HGF was detected using ELISA, and the HGF and c-MET signaling pathway was assessed with Western blot. The proliferation and invasion of human cervical cancer cell lines (HeLa and CaSki cells) were measured using CCK-8 and transwell assays. A HeLa xenograft mouse model was established to determine the effect of ADSCs on tumor growth in vivo.

Results: ADSCs secreted a high level of HGF into the supernatant, while co-culture of ADSCs and cervical cancer cells increased the supernatant level of HGF. The HGF/c-MET pathway was activated in HeLa and CaSki cells co-cultured with ADSCs. Both co-culture with ADSCs and use of ADSC-derived conditioned medium (ADSCs-CM) significantly promoted the proliferation and invasion of cervical cancer cells in vitro, an effect that was reduced by inhibiting tumor cell c-MET expression. Furthermore, ADSCs-CM promoted HeLa cervical tumor growth in vivo, which could be suppressed by intratumoral c-MET siRNA injection.

Conclusion: ADSCs promote cervical cancer growth and invasion through paracrine secretion of HGF and involvement of the HGF/c-MET signaling pathway.

Keywords: ADSCs, cervical cancer, paracrine, proliferation, HGF, c-MET

\section{Introduction}

Cervical cancer is the fourth leading worldwide cause of cancer death in women ${ }^{1}$ and imposes an enormous healthcare burden. Although there has been great progress in cervical cancer prevention, diagnosis, and therapy, treatment outcomes for patients with advanced metastatic cervical neoplasia remain poor. Patients may present with advanced disease due to insufficiently effective screening or inadequate therapeutic strategies. ${ }^{2}$ Cervical cancer arises through a continuous process of transition from dysplastic to neoplastic invasive cancer. Because the etiology of cervical cancer has not been fully elucidated, it is still essential to focus on molecular mechanisms driving its occurrence and progression.
Department of Epidemiology, School of Public Health, Nanjing Medical University, I0I Longmian Road, Nanjing 2III66, People's Republic of China Email rongbinyu@yeah.net

Xiaowei Xi

Department of Obstetrics and Gynecology, Shanghai General Hospital of Nanjing Medical University, Shanghai 200080, People's Republic of China Email xixiaoweil966@I63.com
Cancer Management and Research 2020:12 | |823-| | 832

11823

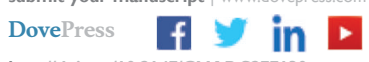

http://doi.org/| 0.21 47/CMAR.S277130 
Hepatocyte growth factor (HGF) can be secreted by tumor cells, stem cells, and stromal cells, and functions in cell reproduction, survival, invasion, tissue development, and organ regeneration through activation of its receptor c-mesenchymal-epithelial transition factor $(\mathrm{c}-\mathrm{MET}){ }^{3}$ C-MET is abnormally expressed in a variety of tumors, including hepatic carcinoma, gastric carcinoma, breast cancer, and cervical cancer. The genetic and epigenetic functions of the HGF/c-MET axis contribute to cancer progression through a variety of mechanisms. ${ }^{4}$ In advanced tumors, $\mathrm{HGF} / \mathrm{c}-\mathrm{MET}$ signaling initiates the invasive growth that promotes cell proliferation, migration, invasion, and angiogenesis. The abnormal HGF/c-MET signaling pathway can promote cervical cancer, while interfering with the HGF/c-MET axis can be used as a therapeutic target for cervical cancer. ${ }^{5}$ For example, verticillin can act as a novel $\mathrm{HGF} / \mathrm{c}-\mathrm{MET}$ inhibitor by suppressing c-MET and downstream FAK/Src signaling pathways through impairment of HGF-induced c-MET phosphorylation. $^{6}$

Adipose-derived stem cells (ADSCs) have attracted a great deal of interest in the stem cell field owing to their capacity for multipotential differentiation towards adipogenic, osteogenic, chondrogenic, and myogenic lineages. Because they are easily obtained from a variety of tissues and have low immunogenicity, ADSCs are ideal seed cells in tissue engineering. ADSCs are a rich source of cytokines and chemokines and can engage in paracrine communication with surrounding cells using HGF, vascular endothelial cell growth factor (VEGF), transforming growth factor (TGF), insulin-like growth factor (IGF), and basic fibroblast growth factor (bFGF). ${ }^{7}$ These cytokines also play pivotal roles in the various physiological functions of ADSCs.

Moreover, when adipocytes become part of the tumor microenvironment (TME), the adipocytes have mechanical and biochemical interactions with tumor cells that can regulate tumor progression. The paracrine cytokines derived from ADSCs within fat tissue make important contributions to the TME and can affect tumor cell proliferation. For example, Chen et al found a relationship between ADSCs and osteosarcoma progression mediated by STAT3 pathway activation in conjunction with changes of MMP-2/9 expression and E-cadherin expression. ${ }^{8} \mathrm{Li}$ et al reported that ADSCs could inhibit the proliferation of urinary bladder tumors in vitro by S-phase arrest and the Wnt/ $\beta$-catenin pathway. ${ }^{9}$ However, it appears that ADSCs may either promote or suppress tumor cell proliferation. For example, Schmid et al confirmed that ADSCs of tumor-adjacent and tumor-distant mammary fat were the main producers in the autotaxin-lysophosphatidic acid axis promoting breast cancer in vitro. ${ }^{10}$ In the in vitro assay with Lewis lung carcinoma cells, ADSCs could enhance the cancer stem cell property and tumor formation capacity through an IL-6 paracrine circuit. ${ }^{11}$ ADSCs-CM was reported to inhibit the growth of liver cancer cells by inhibiting cell proliferation and promoting cell apoptosis, as well as by suppressing cell motility, adhesive capacity, migration and invasion. ${ }^{12}$

Because the roles of ADSCs in cervical carcinoma are still controversial, their clinical use may constitute a safety hazard. This inspired us to explore the relationship between ADSCs and cervical carcinoma. The purpose of the current study was to investigate the effects of ADSC-derived HGF on the growth of cervical cancer cells and further to explore the underlying HGF/c-MET mechanisms. For this purpose, we obtained and cultured ADSCs and collected conditioned medium derived from the ADSCs (ADSCs-CM) to determine how the cells and their conditioned medium affected cell proliferation and invasion of two cervical cancer cell lines in vitro. C-MET siRNA was then used to investigate the participation of the HGF/c-MET axis in cell proliferation and invasion. Finally, the impact of c-MET on in vivo cervical cancer tumor development with ADSCs-CM treatment was evaluated. Our findings show that, through HGF paracrine function, ADSCs possess the ability to promote proliferation and invasion in cervical cancer mediated by activating the HGF/c-MET pathway.

\section{Materials and Methods \\ Cell Lines and Cell Culture}

Human cervical cancer HeLa and CaSki cells were obtained from the American Type Culture Collection (ATCC, USA). These cell lines were cultured in a humidified incubator at $37^{\circ} \mathrm{C}$ in $5 \% \mathrm{CO}_{2}$ and routinely maintained in DMEM supplemented with $10 \%$ FBS, $1 \%$ penicillin $\mathrm{G}$, and $1 \%$ streptomycin. The cultural supernatants of tumor cells and of ADSCs were separately collected, centrifuged, and stored at $-20^{\circ} \mathrm{C}$.

\section{Ethical Approval}

This study was approved by the Ethics Committee of the Women's Hospital of Nanjing Medical University and conducted in accordance with the Declaration of Helsinki. All procedures involving human participants were in accordance with the ethical standards of the 
research committee and its ethical standards. All patients signed an informed consent from before participation.

\section{ADSCs Preparation, Culture, and Identification}

ADSCs were isolated from the liposuction specimens of three healthy, female donors who underwent liposuction surgery for cosmetic purposes, the isolation procedure was performed as described previously. ${ }^{13}$ Briefly, the adipose tissue was carefully washed in $1 \%$ sterile BSA/PBS buffer, cut into tiny pieces, and digested enzymatically with $0.1 \%$ collagenase I (\#C0130, Sigma) in DMEM for 60 min under constant gentle shaking at $37^{\circ} \mathrm{C}$. After dissociation and centrifugation, the sedimented cells were resuspended in DMEM with $10 \%$ FBS and then filtered through a $100 \mu \mathrm{m}$ mesh filter to remove debris. After erythrocyte lysis, the cells were washed twice and seeded into expansion medium at a density of $2 \times 10^{4} /$ $\mathrm{cm}^{2}$. After $24 \mathrm{~h}$, the medium was changed to remove nonadherent cells. Cells at passages 3-5 were acquired for experiments. To identify ADSC, adipogenesis was induced by adipogenic medium (HUXMA-90,031, Cyagen) for two weeks, followed by formalin fixation and incubation in $2 \%$ (wt/vol) Oil Red O reagent for $5 \mathrm{~min}$. Stained cells were photographed for observation of lipid droplets. Flow cytometry was used to analyze the phenotype of ADSCs after incubation with fluorescent-labeled monoclonal antibodies against CD29, CD31, CD90, and CD105 (Biolegend, USA). Finally, the adipogenesis-associated genes leptin and PPAR- $\gamma$ were detected by RT-qPCR. The ADSCs-CM was collected from culture supernatant after no more than five passages of ADSCs, centrifuged at $1000 \times \mathrm{g}$ for $10 \mathrm{~min}$, filtered through $0.22 \mu \mathrm{m}$ filters, and stored at $-20^{\circ} \mathrm{C}$.

\section{RT-qPCR}

Total RNA was isolated using TRIzol Reagent (Invitrogen, Carlsbad, CA, USA), and first-strand cDNA was synthesized using the HiScript II Q RT SuperMix (\#R223-01, Vazyme, Nanjing, China). Complementary DNA and HiScript II One Step qRT-PCR SYBR Green Kit (Q22101, Vazyme, Nanjing, China) were used for real-time PCR.
The resulting data were normalized to GAPDH. Realtime PCR and data collection were performed on an QuantStudio $^{\mathrm{TM}} 5$ instrument (Applied Biosystems, CA, USA). The primers used are as Table 1.

\section{Cell Proliferation Assay}

Cervical cancer cell proliferation was measured using the CCK-8 assay (Beyotime Biotechnology, China) according to the manufacturer's instructions. c-MET siRNA or si-NC transfected cancer cells were seeded into a 96-well plate for attachment. For the CCK-8 assay, the cells were cultured with ADSCs-CM for $0,12,24,36$, and $48 \mathrm{~h}$. At the end of each time point, $10 \mu \mathrm{L}$ of CCK-8 reagent (Dojindo, Japan) was added per well for $4 \mathrm{~h}$. The optical density was measured at $450 \mathrm{~nm}$ using a microplate reader (Molecular Devices, USA).

\section{Transwell Invasion Assay}

The invasion ability of the cancer cells was performed using the transwell assay in 24-well plates (Corning, USA). Cancer cells were seeded in the upper chambers coated with Matrigel. ADSCs or $500 \mu \mathrm{L}$ ADSCs-CM were placed in the lower chamber. The cells were allowed to invade the Matrigel for $24 \mathrm{~h}$. The invasive ability was determined by counting the transmigrated cells stained with crystal violet. The figures were obtained in five random fields in each group by a fluorescent microscope (IX73, Olympus Corp, Japan).

\section{siRNA Transfection}

The c-MET-targeted siRNAs and negative control mismatch sequences were synthesized by Sangon Biotechnology Co., Ltd (China) using the sense and antisense strands. HeLa and CaSki cells were seeded into the petri dish at a density of $6 \times$ $10^{5}$ for $24 \mathrm{~h}$. After cell adherence, siRNA transfection into the HeLa and CaSki cells was performed according to the manufacturer's instructions using Lipofectamine ${ }^{\mathrm{TM}} 2000$ (Invitrogen, USA).

\section{HGF Detection}

Cell culture supernatants were collected after 2 days of culture from HeLa, CaSki, and ADSCs, as well as from transwell co-cultures of ADSCs and either HeLa cells or

Table I Primer Sequences Used for RT-qPCR

\begin{tabular}{|l|l|l|}
\hline Genes & Forward $\left(\mathbf{5}^{\prime} \mathbf{- 3} \mathbf{\prime}\right)$ & Reverse $\mathbf{( 5}^{\prime} \mathbf{-} \mathbf{3}^{\prime} \mathbf{)}$ \\
\hline Leptin & TGCCTTCCAGAAACGTGATCC & CTCTGTGGAGTAGCCTGAAGC \\
PPAR- $\gamma$ & GATGCCAGCGACTTTGACTC & ACCCACGTCATCTTCAGGGA \\
GAPDH & TGTGGGCATCAATGGATTTGG & ACACCATGTATTCCGGGTCAAT \\
\hline
\end{tabular}


CaSki cells. HGF in the supernatants of HeLa or CaSki cells co-cultured with ADSCs was also measured. The concentration of $\mathrm{HGF}$ in ADSCs-CM was measured using a human HGF quantitative ELISA kit (RD Systems, USA). Pre-coated ELISA plates were incubated with $50 \mu \mathrm{L}$ conditioned medium for $2 \mathrm{~h}$ at room temperature. Subsequently, after $200 \mu \mathrm{L}$ conjugate addition, the plate was covered with a new sealer and incubated at room temperature for $2 \mathrm{~h}$. After washing, $200 \mu \mathrm{L}$ substrate solution was then added to each well and incubated at room temperature for $30 \mathrm{~min}$. Finally, $50 \mu \mathrm{L}$ of stop solution was added to each well. The optical density was read at $450 \mathrm{~nm}$ within $30 \mathrm{~min}$. The standard curve was acquired and the concentration of HGF was calculated in triplicate and indicated as $\mathrm{pg} / \mathrm{mL}$.

\section{Western Blotting}

The cells were lysed on ice using cell lysis buffer containing protease inhibitors. Equivalent amounts of protein extract were separated by denaturing SDS-PAGE and electrotransferred onto polyvinylidene fluoride membranes. The membranes were blocked with 5\% non-fat milk in Tris-buffered saline (TBS) for $2 \mathrm{~h}$ and then incubated with anti-c-MET (\#8198), anti-phospho-c-Met (\#3077), bcl-2 (\#3498), bax (\#5023), or anti- $\beta$-actin (\#3700, Cell Signaling Technology, USA) at $4^{\circ} \mathrm{C}$ overnight. The membranes were then incubated with anti-rabbit secondary antibodies for $1 \mathrm{~h}$, after which we detected and quantified the band of protein-antibody complexes using a chemiluminescence detection system (Bio-Rad, USA).

\section{Cervical Cancer Xenograft Mouse Model}

Animal experiments were carried out according to the guidelines of the Nanjing Medical University with approved number: NJMU-KY-20,180,064. The experiments were performed in accordance with the National Guidelines for Experimental Animal Welfare (the Ministry of Science and Technology, China). Six-weekold BALB/c nude mice were purchased from Charles River Laboratories (Beijing, China). The cervical cancer xenograft was established by the subcutaneous injection of HeLa cells $\left(1 \times 10^{7}\right.$ cells suspended in $200 \mu \mathrm{L}$ PBS $)$ into the front flank of the mice as previously reported. ${ }^{14}$ After tumor volume reached about $100 \mathrm{~mm}^{3}$ post-tumor implantation, the mice were divided into 4 groups. These were denoted as control group (no conditioned medium injection), ADSCs-CM group, Lipo + ADSCs-CM group and siRNA + ADSCs-CM group. ADSC-CM was injected into the tumor once per week. Liposome with negative control or c-MET siRNA was injected into the tumor once per week. The mice were monitored weekly for tumor growth and tumor volume detection, tumor volume (V) was calculated by tumor length (L) and width $(\mathrm{W})$ as: $\mathrm{V}=\mathrm{L} \times \mathrm{W}^{2}$ / 2. At 21 days post-treatment, the mice were sacrificed, and the tumors were isolated for photography and weighing. Hematoxylin and eosin (H\&E) staining and immunofluorescence (IF) of the tumors were investigated to analyze the pathological feature and c-MET expression. The apoptosis-related proteins $\mathrm{Bcl}$ and $\mathrm{Bax}$ were evaluated by Western blotting as described above.

\section{Immunofluorescence (IF)}

Tissue sections were deparaffinized and rehydrated, and antigen retrieval was conducted following the manufacturer's instructions. Endogenous peroxidase activity was blocked with $0.3 \%$ hydrogen peroxide for $15 \mathrm{~min}$. Slides were then blocked with goat serum, avidin solution and biotin solution. The slides were incubated with rabbit anti-human polyclonal antibodies against c-MET (1:200 dilution, Proteintech) at $4{ }^{\circ} \mathrm{C}$ overnight and then probed with AlexaFlour 594-conjugated secondary antibody (Invitrogen) and mounted with Vectashield containing DAPI (Vector Laboratories, Burlingame, CA). Images were acquired on a Zeiss LSM 880 confocal laser scanning microscope (Carl Zeiss MicroImaging, Inc, Thornwood, NY).

\section{Statistical Analysis}

All data were expressed as the means \pm standard deviation (SD) and were obtained from at least three independent experiments. Statistical testing was carried out with a twotailed Student's $t$-test or a one-way analysis of variance. A value of $\mathrm{P}<0.05$ was regarded as statistically significant.

\section{Results}

\section{The Characteristics of ADSCs}

$\mathrm{CD}$ markers of ADSCs were characterized by flow cytometry. The ADSCs were positive for mesenchymal cell markers including CD29, CD105, and CD90, and negative for the endothelial cell-specific marker CD31 (Figure 1A). Microscopic observation showed that ADSCs exhibited a fibroblast-like cell morphology and formed a monolayer (Figure 1B). Oil Red $\mathrm{O}$ staining showed the formation of lipid droplets in ADSCs, confirming their potential for adipogenic differentiation (Figure 1C). Furthermore, the fat-related 
A

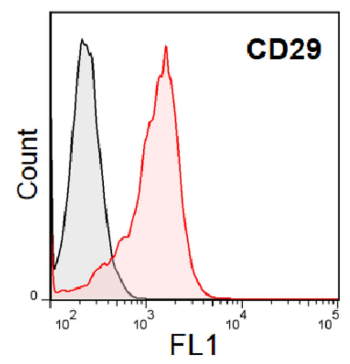

B

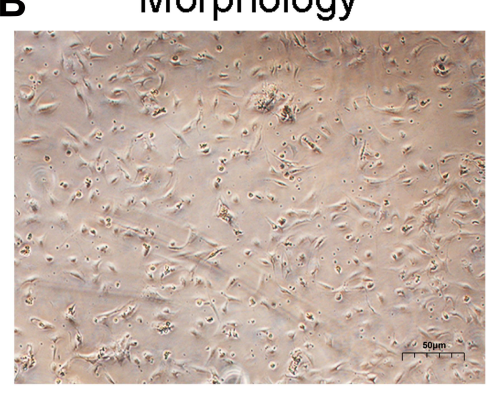

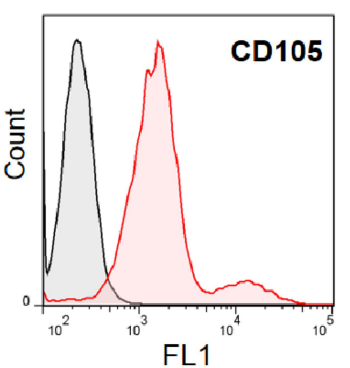
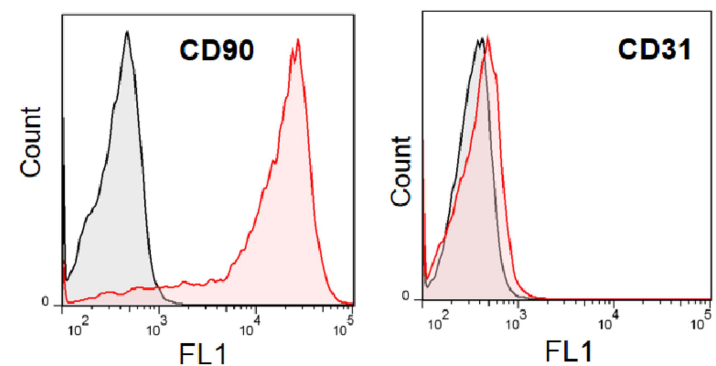

FL1
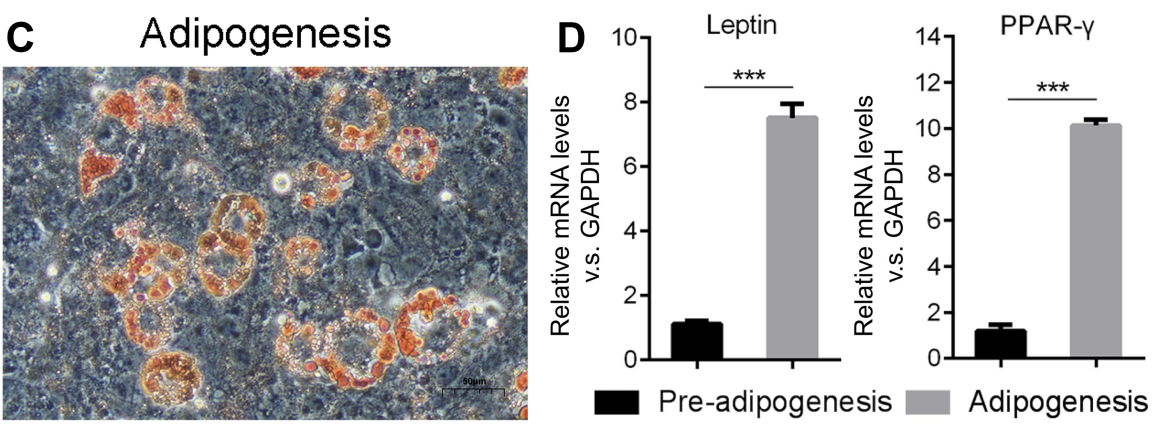

Figure I ADSC identifications. (A) CD29, CD 105, CD90, and CD31 in ADSCs as characterized by flow cytometry. Grey represents the negative control, red represents the positive cells. (B) Cell morphology for ADSCs after 3 passages. (C) The adipogenic differentiation potential of ADSCs demonstrated by Oil Red O staining. Scale bar $=50 \mu \mathrm{m}$. (D) The relative mRNA level of leptin and PPAR- $\gamma$ detected by RT-PCR. $* * * P<0.001$.

A

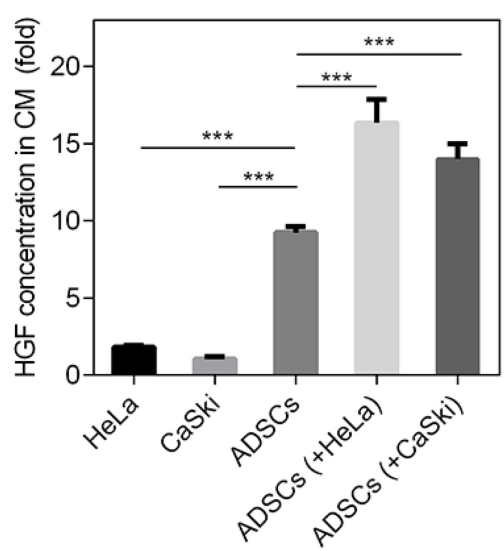

D

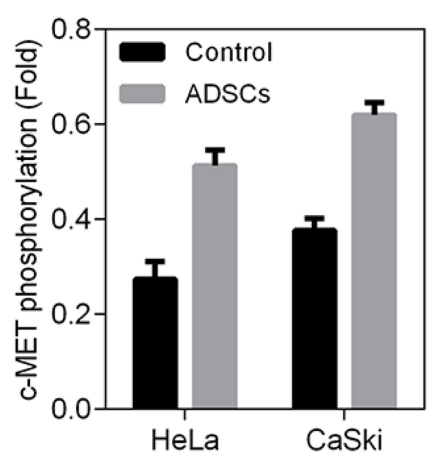

B

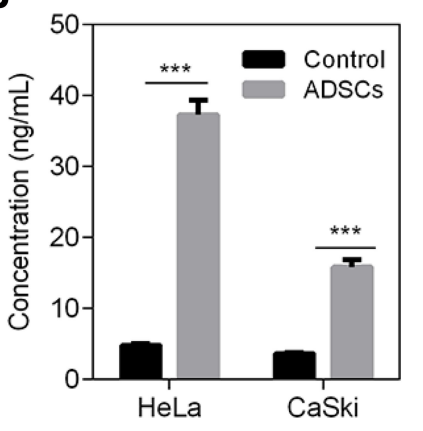

E

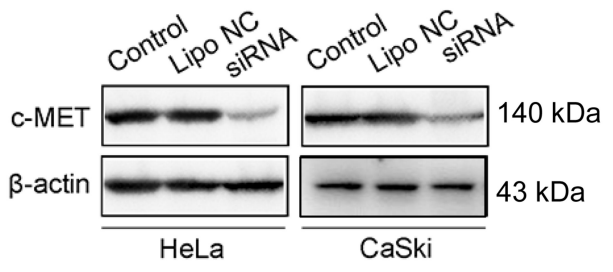

C

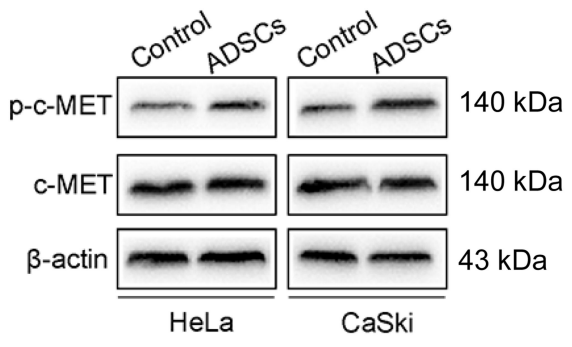

$\mathbf{F}$

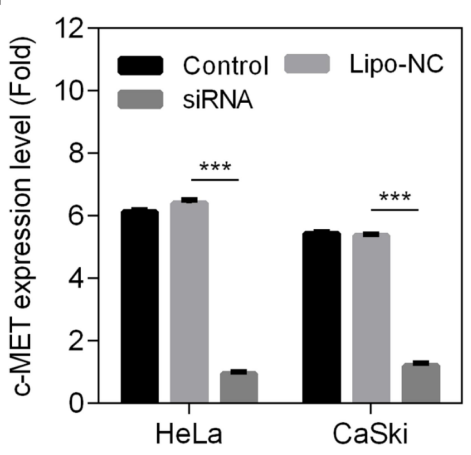

Figure 2 HGF/c-MET activation. (A) The HGF relative level in supernatants of HeLa, CaSki, ADSCs, and ADSCs co-cultured with either HeLa or CaSki cells. (B) HGF concentration in the supernatants of HeLa or CaSki cells co-cultured with ADSCs determined by ELISA assay. (C) The protein expression of c-MET and p-c-MET detected by Western blot. (D) The phosphorylation ratio of c-MET quantified using Image J software. (E and $\mathbf{F})$ The siRNA transfection effect in the HeLa or CaSki cells detected by Western blotting. $* * * P<0.001$. 
genes leptin and PPAR- $\gamma$ were significantly elevated in ADSCs after adipogenesis (Figure 1D). These findings confirmed that the ADSCs were successfully obtained and could be utilized for subsequent experiments.

\section{HGF Expression in ADSCs and HGF/ c-MET Change in Cervical Cancer Cells}

ELISA assay of HGF expression in culture supernatant showed that the ADSCs secreted more HGF than HeLa and CaSki cells, while ADSCs co-cultured with HeLa or CaSki secreted higher amounts of HGF than ADSCs cultured alone (Figure 2A). Similarly, HGF in the supernatants of HeLa or CaSki cells co-cultured with ADSCs were also significantly elevated compared with the normally cultured control group (Figure 2B). Thus, ADSCs secreted abundant HGF attributable to their paracrine function, and the interaction of ADSCs and tumor cells stimulated the secretion of HGF both in ADSCs and tumor cells. Because HGF is an important cytokine derived from ADSCs and the HGF/c-MET is involved in tumor progression, we investigated c-MET activation. Western blotting showed upregulation of the ratio of c-MET phosphorylation in co-cultured to control cancer cell lines (Figure 2C and D). To further explore the mechanism of ADSCs on tumor cells, c-MET siRNA was transfected into Hela and CaSki cells to down-regulate their c-MET expression. C-MET siRNA significantly reduced the c-MET protein as confirmed by Western blotting (Figure 2E and F).

\section{ADSCs Enhances Proliferation and Invasion of Cervical Cancer Cells by}

\section{Paracrine in vitro}

To detect the impact of ADSCs on proliferation and invasion, Hela and CaSki cells were cultured with ADSCs-CM or co-cultured with ADSCs (co-ADSCs). Conventionally cultured cells were used as controls. The CCK-8 assay showed higher cell proliferation rates when
A

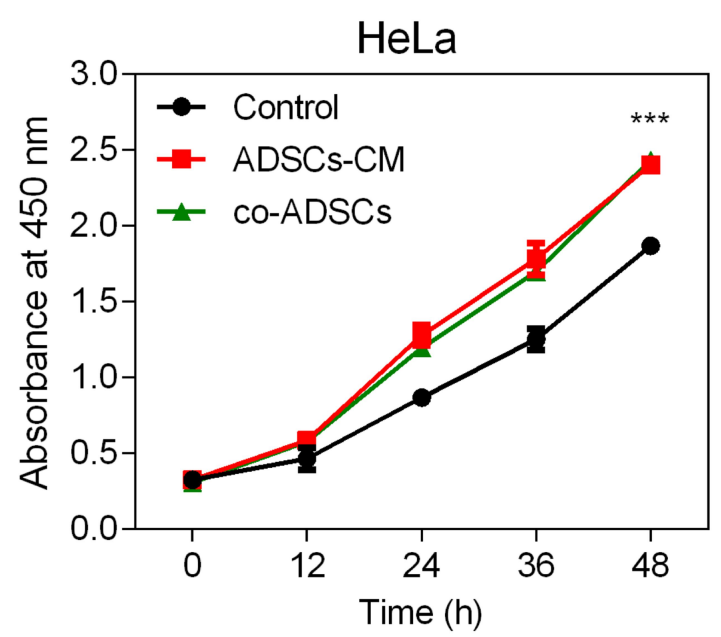

C

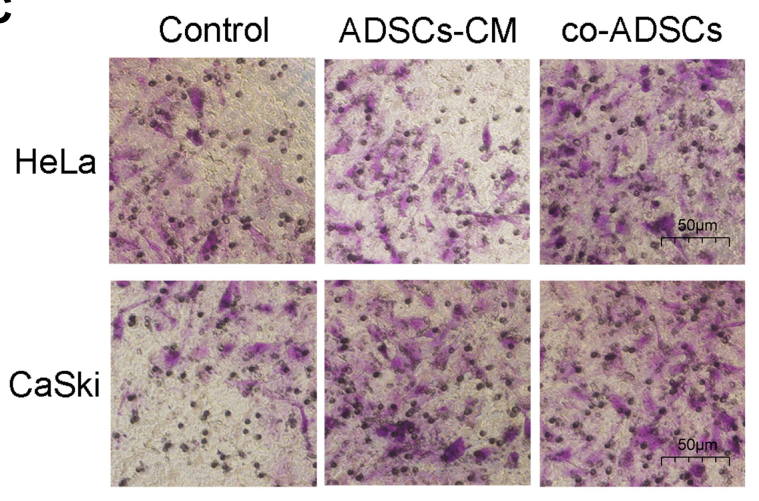

B

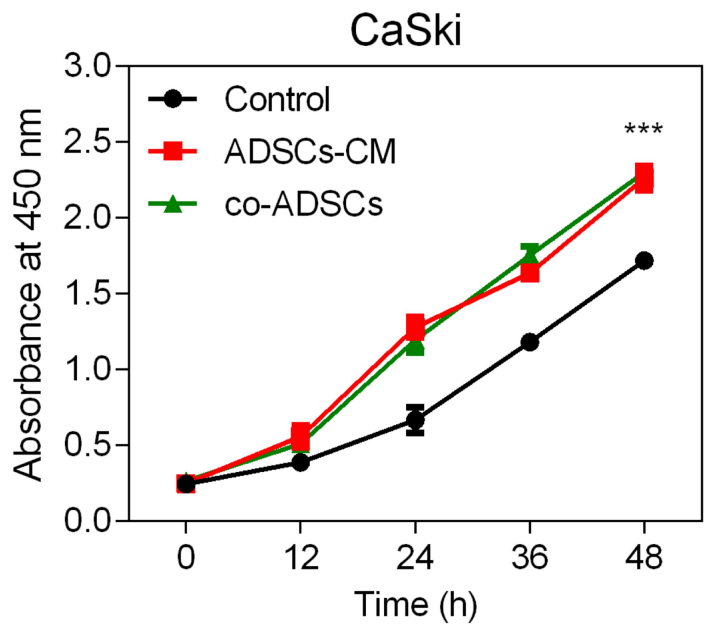

D

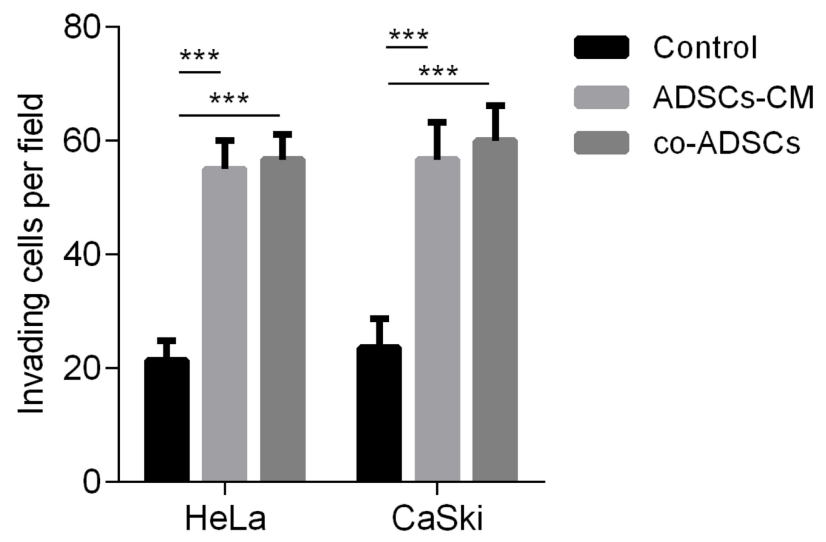

Figure 3 The effect of ADSCs-derived conditioned medium (ADSCs-CM) or co-culture of ADSCs on tumor proliferation and invasion. The proliferative activity of HeLa (A) and CaSki (B) cells detected by CCK-8. (C) Transmigrated cells were fixed and stained with crystal violet in the transwell assay. (D) Stained cells were counted in five randomly selected microscopic fields. Scale bar $=50 \mu \mathrm{m}$. $* * * P<0.001$. 
incubated with either ADSCs-CM or co-ADSCs compared with control (Figure $3 \mathrm{~A}$ and $\mathrm{B}$ ). The transwell invasion assay also showed a significantly increased number of invading cancer cells with crystal violet staining in ADSCs-CM or co-ADSCs group compared with the control (Figure 3C and D). However, there was no difference in cell proliferation and invasion between ADSCs-CM and co-ADSCs groups. These results indicate that ADSCs-CM and co-ADSCs could enhance cervical cancer cell proliferation and invasion in vitro, further emphasizing the importance of paracrine signaling by ADSCs.

\section{c-MET Inhibition Reduces the Ability of ADSC to Promote Proliferation and Invasion of Tumor Cells}

In the cell proliferation assay, the ADSCs-CM and Lipo + ADSCs-CM group exhibited increased proliferative activity relative to control, while the siRNA + ADSCs-CM group exhibited lower tumor proliferative activity (Figure 4A and $\mathrm{B}$ ). In the invasion assay, the ADSCs-CM and Lipo +
ADSCs-CM groups exhibited up-regulated invasion compared with the control group, while the siRNA + ADSCs$\mathrm{CM}$ group exhibited lower invasion (Figure $4 \mathrm{C}$ and $\mathrm{D}$ ), indicating that c-MET inhibition in the ADSCs-CM + c-MET siRNA group resulted in the downregulation of invasion ability. Therefore, c-MET siRNA attenuated the proliferation and invasion ability of Hela and CaSki cells, suggesting that ADSCs-CM facilitation was mediated through the HGF/c-MET signal pathway.

\section{ADSCs Promote HeLa Xenograft Growth by Targeting the HGF/c-MET Pathway Through Paracrine Secretion}

Next, we investigated the effect of ADSCs-CM on HeLa cell xenograft growth in vivo. The tumor volumes in each group were measured and plotted as a function of time (Figure 5A). Tumors in the control group grew rapidly, but the tumors grew more rapidly in the ADSCs-CM and Lipo + ADSCs-CM injected groups, as emphasized in the photograph of isolated tumors (Figure 5B). In contrast,
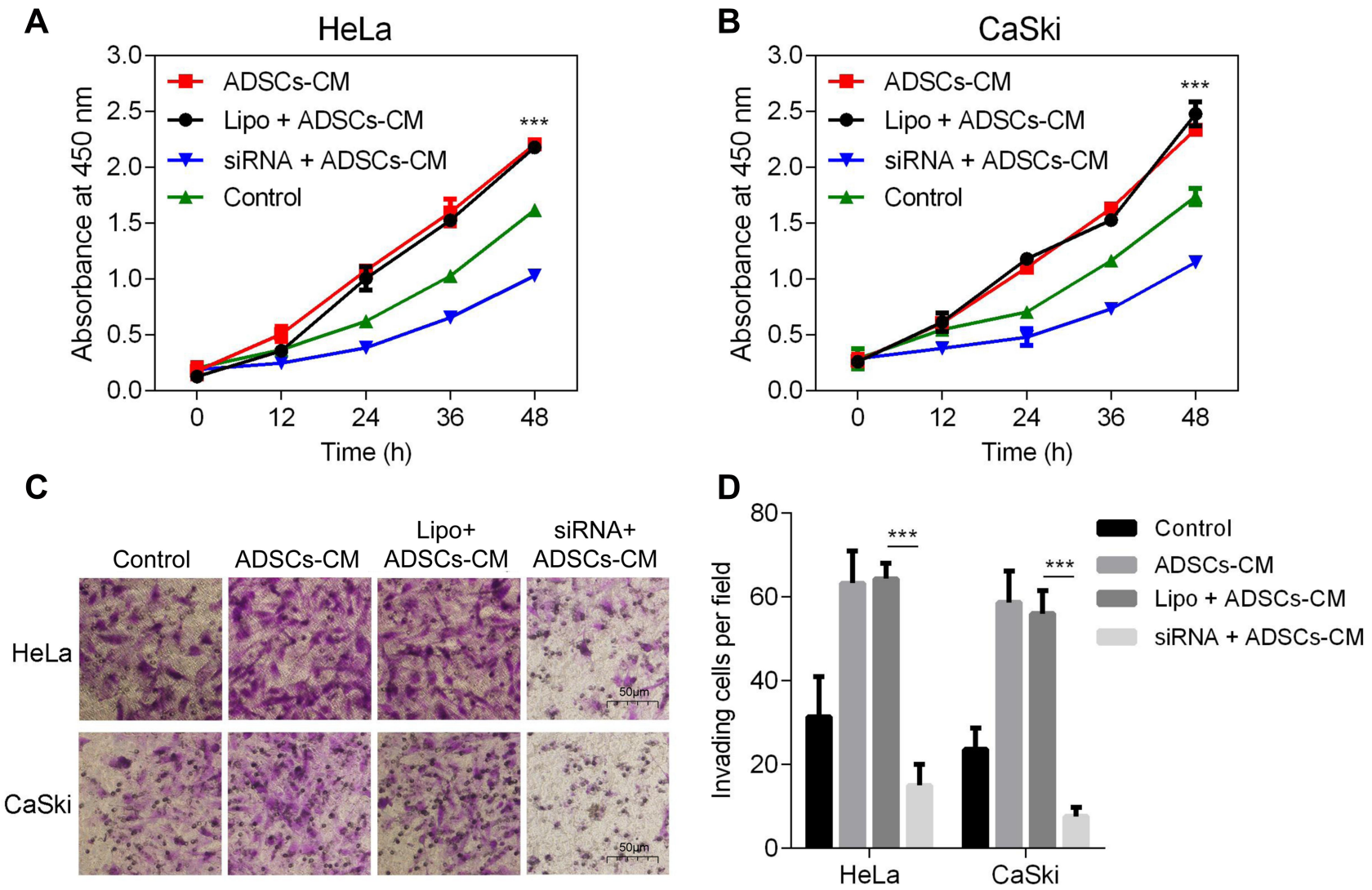

Figure 4 Inhibition of c-MET represses the ADSCs' ability to promote tumor proliferation and invasion ability. The proliferation activity of Hela (A) and CaSki (B) cells detected by CCK-8 in PBS, ADSCs-CM group, Lipo + ADSCs-CM group and siRNA + ADSCs-CM group. (C) The transmigrated cells in the above four groups by transwell assay. (D) Quantitative cell staining with crystal violet, scale bar $=50 \mu \mathrm{m}$. $* * * P<0.00 \mathrm{I}$. 

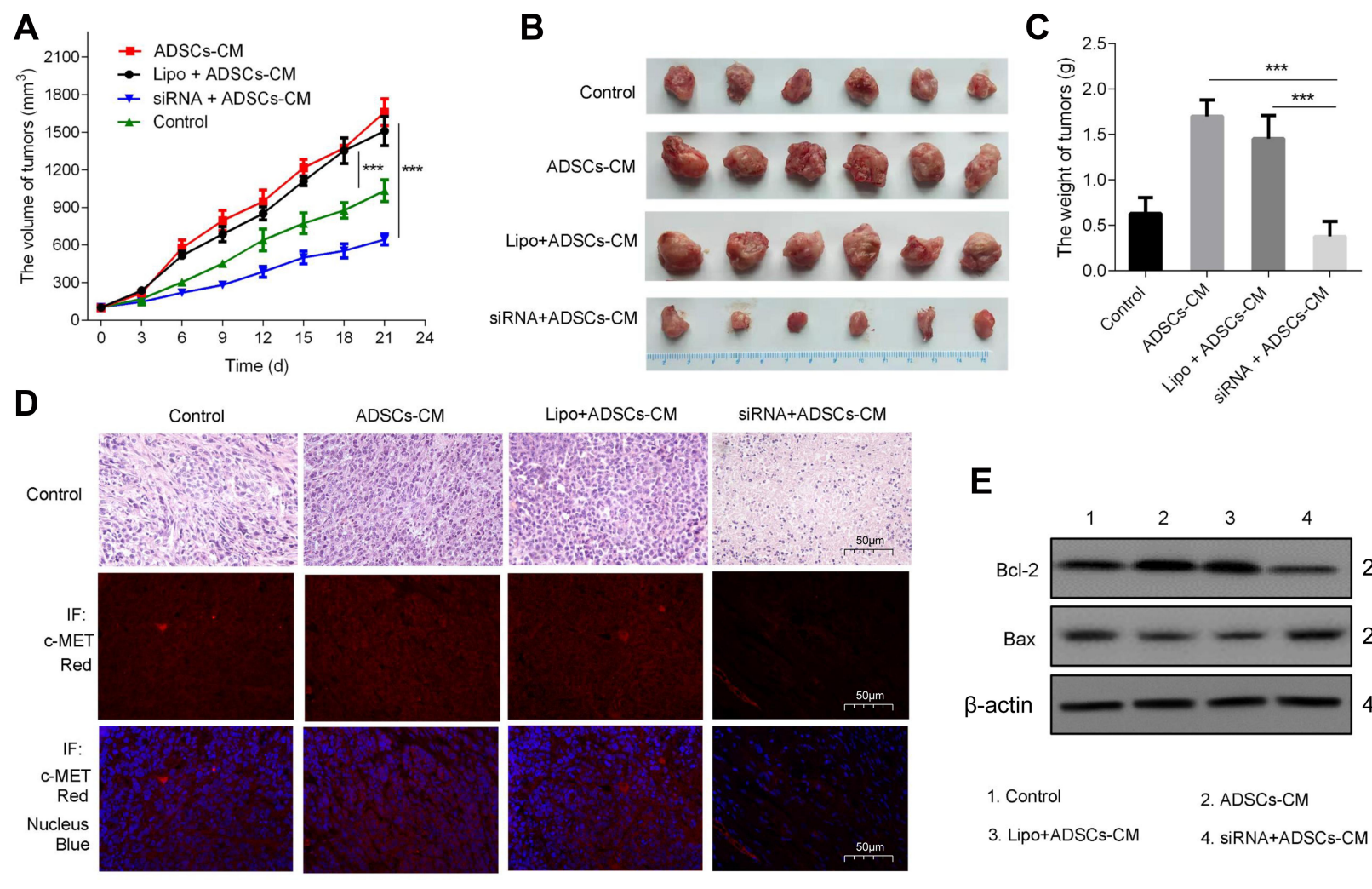

E

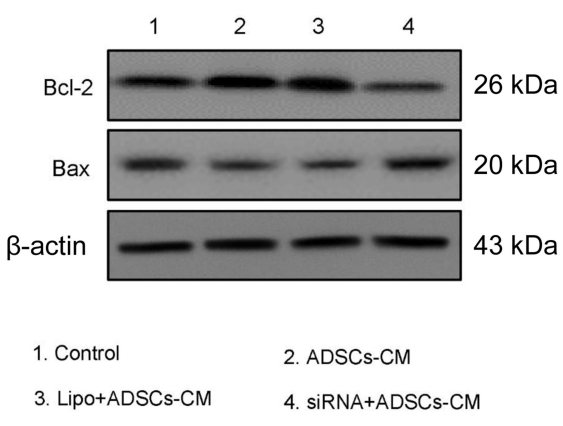

Figure 5 Effects of ADSCs on cervical HeLa tumor growth in PBS, ADSCs-CM group, Lipo + ADSCs-CM group and siRNA + ADSCs-CM group. (A) Tumor volume (mm $\left.{ }^{3}\right)$ measurement every 3 days for $2 \mathrm{I}$ days. (B) Representative photograph of isolated tumors at the end of treatment. (C) Tumor weight (g) in each group. (D) H\&E and immunofluorescence staining of tumor biopsies. The c-MET protein was marked as red and the nucleus was marked as blue. The images were observed by light microscopy and fluorescence microscope, scale bar $=50 \mu \mathrm{m}$. (E) The expression of apoptosis-associated proteins Bcl-2 and Bax detected by Western blotting. $* * * P<0.00 \mathrm{I}$.

when simultaneously treated with ADSCs-CM plus c-MET siRNA, the mice exhibited obviously decreased tumor volumes and weights compared with the ADSCs$\mathrm{CM}$ and control groups (Figure 5C). Immunofluorescence results confirmed that c-MET expression was seriously weakened (as marked in red) due to c-MET siRNA treatment (Figure 5D lower). Western blot analysis revealed a significant upregulation of $\mathrm{Bcl}-2 / \mathrm{Bax}$ expression ratio in ADSCs-CM and Lipo + ADSCs-CM groups compared with control group, and c-Met siRNA treatment alleviated this upregulation significantly (Figure 5E). These in vivo results indicate that the $\mathrm{HGF}$-containing ADSCs-CM might play an important role in cervical tumor promotion through the HGF/c-MET signal pathway.

\section{Discussion}

In recent years, because adipose tissue is easily accessible and is the most abundant source of adult stem cells, with the ability to differentiate into myriad lineages with paracrine expression of cytokine growth factors, ADSCs have been considered as a promising tool for disease therapy (including oncotherapy) and regenerative medicine. ${ }^{15}$ The paracrine cytokines CXCL1 and CXCL8 derived from ADSCs in tumor-associated fat tissue make important contributions to the TME and can affect the features of tumor cells. ${ }^{16}$ For example, Wang reported that ADSCs could enhance ovarian cancer growth and metastasis by increasing thymosin beta $4 x$-linked expression. ${ }^{17}$ It was also reported that ADSCs could attenuate the cisplatininduced apoptosis in ovarian cancer cells, serving as a therapeutic method. ${ }^{18}$ On the other hand, Takahara showed that ADSCs inhibited prostate cancer cell proliferation by inducing apoptosis. ${ }^{19}$ Thus, it appears that ADSCs can either promote or inhibit tumor growth.

One possible reason for the disparate results might be that the effect of ADSC-tumor interaction is different between in vitro and in vivo experiments. In addition, fat distribution, function, and ADSC characteristics are different in different tumor types. Furthermore, due to differences in fat tissue environments, the cytokine expression profile of ADSCs may show a significant difference. ${ }^{20}$ Any of the above factors may have some relationship to the reciprocal effects. As reported by Lu et al, ADSCs played a pro-malignant role in tumor development of Lewis lung carcinoma cells by promoting cancer stem cell properties through the IL-6 paracrine 


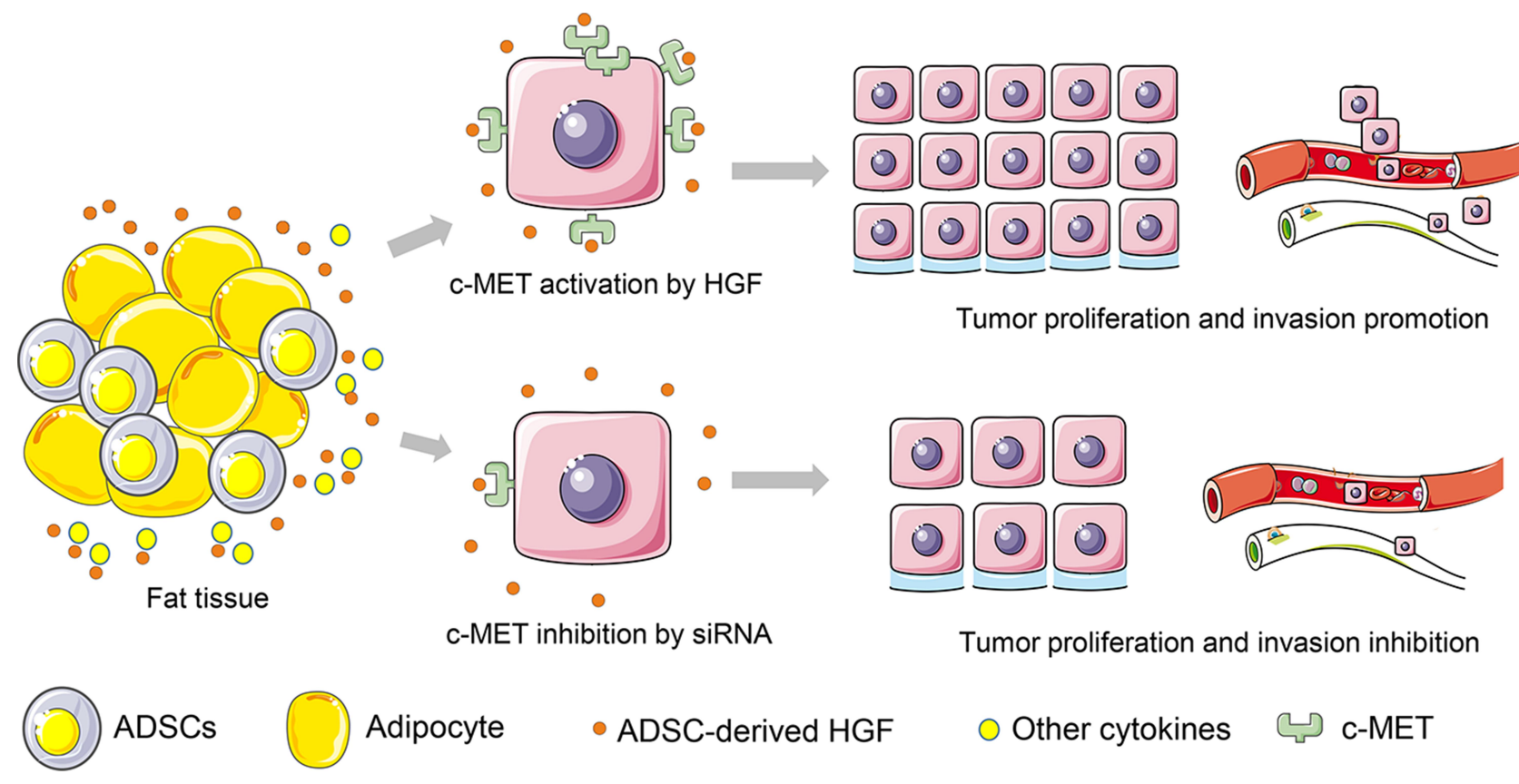

Figure 6 Schematic illustration. HGF is a very important cytokine derived from ADSCs by paracrine function. The interaction of ADSCs and tumor cells could promote the HGF secretion in ADSCs and tumor cells. When co-cultured with ADSCs, the HGF/c-MET pathway could be activated in cervical cancer cells, resulting in a more proliferative and invasive phenotype of cervical cancer. Once treated with c-MET siRNA to inhibit the c-MET protein expression, the tumor proliferative and invasive ability induced by ADSCs could be repressed.

circuit, which is important for safety considerations in the clinic. ${ }^{11}$ In this study, we successfully isolated and identified human ADSCs and assessed their effects on cervical cancer cell growth. The results showed that ADSCs could secrete HGF and obviously promote the proliferation and invasion of cervical cancer both in vitro and in vivo, supporting evidence of tumor promotion. ADSCs located in the surrounding tumor tissue play a key role in the modulation of the tumor microenvironment. ${ }^{21}$ In vivo, the ADSCs might be recruited to the tumor and then affect cancer cell viability.

HGF and c-MET overexpression and activation are important indicators for cervical cancer progression, and may be considered as prognostic, predictive, or therapeutic biomarkers in cervical cancer patients. ${ }^{22}$ The HGF/c-MET axis induces c-MET phosphorylation to activate downstream signaling molecules, including PI3K, STAT3, Grb2, and Gab1. ${ }^{23}$ Here, we found that the in vitro and in vivo tumorpromoting effects of ADSCs were mediated at least in part by the paracrine secretion HGF (Figure 6). The related mechanism involves c-MET phosphorylation in the HGF/ c-MET signaling pathway. It is conceivable that increased levels of HGF derived from ADSCs had a significant proliferative effect on cervical cancer. In that case, suppressing the HGF/c-MET pathway might be a potential therapeutic strategy for cervical cancer. Paracrine cytokine secretion
ADSCs, represented by HGF, plays a part in the tumor growth environment. For those reasons, the clinical application of ADSCs in tumor therapy requires special consideration. The complex interactions between ADSCs, the TME, and tumor progression demands more research.

Here, we demonstrated that ADSCs promote the proliferation and invasion of cervical cancer cells in vitro. Furthermore, ADSCs with paracrine function promote cervical tumor growth in vivo. These tumor-promoting effects appear to be partially mediated by ADSC-derived HGF and activation of the $\mathrm{HGF} / \mathrm{c}-\mathrm{MET}$ signaling pathway. Paracrine cytokine secretion from ADSCs, exemplified by HGF, makes an important contribution to the TME. Further studies are needed to improve our understanding of the complex interactions between ADSCs and cervical cancer.

\section{Data Sharing Statement}

The datasets used and/or analyzed during the present study are available from the corresponding author on reasonable request.

\section{Ethics Approval and Informed Consent}

This work was conducted under the support of the Ethics Committee of Women's Hospital of Nanjing Medical University. 


\section{Acknowledgment}

This work was supported by Nanjing Medical Science and Technique Development Foundation (YKK18157).

\section{Disclosure}

Yongning Zhai and Wangfei Wu are co-first authors for this study. The authors declare that there is no conflicts of interest regarding the publication of this paper.

\section{References}

1. Bray F, Ferlay J, Soerjomataram I, Siegel RL, Torre LA, Jemal A. Global cancer statistics 2018: GLOBOCAN estimates of incidence and mortality worldwide for 36 cancers in 185 countries. CA Cancer J Clin. 2018;68(6):394-424. doi:10.3322/caac.21492.

2. Rodrigues C, Joy LR, Sachithanandan SP, Krishna S. Notch signalling in cervical cancer. Exp Cell Res. 2019;385(2):111682. doi:10.1016/j.yexcr.2019.111682.

3. Owusu BY, Galemmo R, Janetka J, Klampfer L. Hepatocyte growth factor, a key tumor-promoting factor in the tumor microenvironment. Cancers. 2017;9:4. doi:10.3390/cancers9040035.

4. Spina A, De Pasquale V, Cerulo G, et al. HGF/c-MET axis in tumor microenvironment and metastasis formation. Biomedicines. 2015;3 (1):71-88. doi:10.3390/biomedicines3010071.

5. Boromand N, Hasanzadeh M, ShahidSales S, et al. Clinical and prognostic value of the C-Met/HGF signaling pathway in cervical cancer. J Cell Physiol. 2018;233(6):4490-4496. doi:10.1002/ jсp.26232.

6. Lu J, Li X, Tu K, Guan Y, Fung KP, Verticillin LF. A suppresses HGF-induced migration and invasion via repression of the c-Met/ FAK/Src pathway in human gastric and cervical cancer cells. Onco Targets Ther. 2019;12:5823-5833. doi:10.2147/OTT.S208683.

7. Kim YW, Min HJ, Choi RJ, Lee DH, Cheon YW. Insulin promotes adipose-derived stem cell differentiation after fat grafting. Plast Reconstr Surg. 2018;142(4):927-938. doi:10.1097/PRS.000000000 0004814.

8. Wang Y, Chu Y, Yue B, et al. Adipose-derived mesenchymal stem cells promote osteosarcoma proliferation and metastasis by activating the STAT3 pathway. Oncotarget. 2017;8(14):23803-23816. doi:10. 18632/oncotarget.15866.

9. Wang T, Yu X, Lin J, et al. Adipose-derived stem cells inhibited the proliferation of bladder tumor cells by s phase arrest and wnt/beta-catenin pathway. Cell Reprogram. 2019;21(6):331-338. doi:10.1089/ cell.2019.0047.

10. Schmid R, Wolf K, Robering JW, et al. ADSCs and adipocytes are the main producers in the autotaxin-lysophosphatidic acid axis of breast cancer and healthy mammary tissue in vitro. BMC Cancer. 2018;18(1):1273. doi:10.1186/s12885-018-5166-z.
11. Lu JH, Wei HJ, Peng BY, et al. Adipose-derived stem cells enhance cancer stem cell property and tumor formation capacity in lewis lung carcinoma cells through an interleukin-6 paracrine circuit. Stem Cells Dev. 2016;25(23):1833-1842. doi:10.1089/scd.2016.0163

12. Xie H, Liao N, Lan F, Cai Z, Liu X, Liu J. 3D-cultured adipose tissue-derived stem cells inhibit liver cancer cell migration and invasion through suppressing epithelial-mesenchymal transition. Int $\mathrm{J} \mathrm{Mol}$ Med. 2018;41(3):1385-1396. doi:10.3892/ijmm.2017.3336.

13. Hu L, Hu J, Zhao J, et al. Side-by-side comparison of the biological characteristics of human umbilical cord and adipose tissue-derived mesenchymal stem cells. Biomed Res Int. 2013;2013:438243. doi: $10.1155 / 2013 / 438243$.

14. Li S, Ma YM, Zheng PS, Zhang P. GDF15 promotes the proliferation of cervical cancer cells by phosphorylating AKT1 and Erk1/2 through the receptor ErbB2. J Exp Clin Cancer Res. 2018;37(1):80. doi:10.1186/s13046-018-0744-0.

15. Bora P, Majumdar AS. Adipose tissue-derived stromal vascular fraction in regenerative medicine: a brief review on biology and translation. Stem Cell Res Ther. 2017;8(1):145. doi:10.1186/s13287017-0598-y.

16. Wang Y, Liu J, Jiang Q, et al. Human adipose-derived mesenchymal stem cell-secreted CXCL1 and CXCL8 facilitate breast tumor growth by promoting angiogenesis. Stem Cells. 2017;35(9):2060-2070. doi:10.1002/stem.2643.

17. Chu Y, You M, Zhang J, et al. adipose-derived mesenchymal stem cells enhance ovarian cancer growth and metastasis by increasing thymosin beta 4X-linked expression. Stem Cells Int. 2019;2019:9037197. doi:10.1155/2019/9037197.

18. Wen Y, Guo Y, Huang Z, Cai J, Wang Z. Adipose-derived mesenchymal stem cells attenuate cisplatin-induced apoptosis in epithelial ovarian cancer cells. Mol Med Rep. 2017;16(6):9587-9592. doi:10. 3892/mmr.2017.7783.

19. Takahara K, Ii M, Inamoto T, et al. Adipose-derived stromal cells inhibit prostate cancer cell proliferation inducing apoptosis. Biochem Biophys Res Commun. 2014;446(4):1102-1107. doi:10.1016/j. bbrc.2014.03.080.

20. Wada Y, Ikemoto T, Morine Y, et al. The differences in the characteristics of insulin-producing cells using human adipose-tissue derived mesenchymal stem cells from subcutaneous and visceral tissues. Sci Rep. 2019;9(1):13204. doi:10.1038/s41598-019-49701-0.

21. Rey F, Lesma E, Massihnia D, et al. Adipose-derived stem cells from fat tissue of breast cancer microenvironment present altered adipogenic differentiation capabilities. Stem Cells Int. 2019;2019:1480314. doi:10.1155/2019/1480314.

22. Peng J, Qi S, Wang P, Li W, Liu C, Li F. Diagnosis and prognostic significance of c-Met in cervical cancer: a meta-analysis. Dis Markers. 2016;2016:6594016. doi:10.1155/2016/6594016.

23. Wu Q, Li B, Li Z, Li J, Sun S, Sun S. Cancer-associated adipocytes: key players in breast cancer progression. J Hematol Oncol. 2019;12 (1):95. doi:10.1186/s13045-019-0778-6.

\section{Publish your work in this journal}

Cancer Management and Research is an international, peer-reviewed open access journal focusing on cancer research and the optimal use of preventative and integrated treatment interventions to achieve improved outcomes, enhanced survival and quality of life for the cancer patient.
The manuscript management system is completely online and includes a very quick and fair peer-review system, which is all easy to use. Visit http://www.dovepress.com/testimonials.php to read real quotes from published authors. 